\title{
Meat consumption is associated with higher dementia prevalence: a cross-sectional analysis of UK Biobank
}

\author{
H. Zhang ${ }^{1}$, L.J. Hardie ${ }^{2}$, D.C. Greenwood ${ }^{3}$ and J.E. Cade ${ }^{1}$ \\ ${ }^{1}$ Nutritional Epidemiology Group, School of Food Science and Nutrition, University of Leeds, Leeds LS2 9JT, UK, \\ ${ }^{2}$ Division of Clinical and Population Sciences, Leeds Institute of Cardiovascular and Metabolic Medicine, School of \\ Medicine, University of Leeds, Leeds LS2 9JT, UK and \\ ${ }^{3}$ Leeds Institute for Data Analytics, University of Leeds, Leeds LS2 9JT, UK
}

Dementia represents an increasing burden of disease in aging societies worldwide; modifiable lifestyle factors, especially diet, have been implicated and represent potential targets for primary prevention ${ }^{(1)}$. Although the role of processed and red meat consumption in relation to certain cancers has been established ${ }^{(2)}$, its involvement in dementia development currently remains debatable, while there is an increasing trend of meat intakes globally.

The intake frequencies per week of total meat comprising red meat, processed meat, and poultry were assessed via a 47 - item food frequency questionnaire in 502493 UK Biobank participants mean age 56.5 years (SD: 8.1) at recruitment between 2006-2010. Prevalent dementia cases were self-reported or hospital- and mortality- registered. Odds ratios (OR) for each meat type in relation to dementia risk were estimated in logistic regression models adjusting for age, sex, ethnicity, Townsend deprivation index, education level, smoking status, alcohol status, body mass index, physical activity level, sleeping hours, stroke history, family history of dementia.

There were 564 prevalent dementia cases mean age 59.8 years (SD: 7.5). Our results show that increased odds of dementia were associated with total-meat consumption by 5\% (adjusted OR: 1.05, 95\% CI: 1.01 to $1.10, P=0.015$ ) for each additional portion of meat/week, processed meat by $14 \%$ (adjusted OR: $1.14,95 \%$ CI: 1.07 to $1.22, P<0.001$ ), and red meat by $9 \%$ (adjusted OR: 1.09 , $95 \%$ CI: 1.02 to $1.16, P=0.017$ ), but not poultry (adjusted OR: $0.94,95 \%$ CI: 0.87 to $1.03, P=0.195$ ). However, sex-specific associations between dementia prevalence and consumption of red meat or total meat were pronounced in men (adjusted OR: 1.11,95\%CI: 1.04 to $1.19, P=0.002$ for red meat; adjusted OR: $1.07,95 \% \mathrm{CI}$ :

1.03 to $1.12, P=0.002$ for total meat), but not in women (adjusted OR: $1.02,95 \%$ CI: 0.86 to $1.21, P=0.783$ for red meat; adjusted OR: $1.02,95 \%$ CI: 0.93 to $1.12, P=0.730$ for total meat).

Our findings suggest that higher frequency of meat consumption, especially processed meat, is associated with greater dementia prevalence, especially among men in the UK. However, due to the cross-sectional nature of this study the direction of effect is uncertain; these associations need to be examined in longitudinal studies further.

\section{References}

1. Kivipelto M, Mangialasche F \& Ngandu T (2018) Nat Rev Neurol 14, 653-666.

2. Bouvard V, Loomis D, Guyton KZ, et al. (2015) Lancet Oncol 16, 1599-1600. 